\title{
Retraction Note: Pre-treatment DWI as a predictor of overall survival in locally advanced pancreatic cancer treated with Cyberknife radiotherapy and sequential S-1 therapy
}

Yu Zhang ${ }^{1+}$, Xiaofei Zhu ${ }^{2+}$, Denghui $\mathrm{Liu}^{3+}$, Jiaqi Song ${ }^{4}$, Huojun Zhang ${ }^{2}$ and Jianping $\mathrm{Lu}^{1 *}$

\section{Retraction note}

The authors have retracted this article because Figures. 2, 3 and 4, as well as parts of the text have been previously published [1]. This article is therefore redundant. Yu Zhang, Denghui Liu, Jiaqi Song, Huojun Zhang and Jianping Lu agree to this retraction. Xiaofei Zhu has not responded to any correspondence from the publisher about this retraction.

\begin{abstract}
Author details
'Department of Radiology, Changhai Hospital Affiliated to the Second Military Medical University, Changhai Road 168, Yangpu district, Shanghai 200433, People's Republic of China. 'Department of Oncology Radiation, Changhai Hospital Affiliated to the Second Military Medical University, Changhai Road 168, Yangpu district, Shanghai 200433, People's Republic of China. ${ }^{3}$ Department of Orthopedics, No. 113 Hospital of People's Liberation Army, East Zhongshan Road 377, Jiangdong District, Ningbo 315000, People's Republic of China. ${ }^{4}$ Department of health statistics, Second Military Medical University, Xiangyin Road 800, Yangpu district, Shanghai 200433,

People's Republic of China.
\end{abstract}

Received: 20 August 2018 Accepted: 20 August 2018

Published online: 04 September 2018

\footnotetext{
Reference

1. Zhang Y, Zhu X, Liu R, Wang X, Sun G, Song J, Lu J, Zhang H. Combination of Pre-Treatment DWI-Signal Intensity and S-1 Treatment: A Predictor of Survival in Patients with Locally Advanced Pancreatic Cancer Receiving Stereotactic Body Radiation Therapy and Sequential S-1. Translational Oncology. 2018;11(2):399-405.
}

\footnotetext{
* Correspondence: LDH870118@163.com

${ }^{\dagger}$ Yu Zhang, Xiaofei Zhu and Denghui Liu contributed equally to this work. 'Department of Radiology, Changhai Hospital Affiliated to the Second Military Medical University, Changhai Road 168, Yangpu district, Shanghai 200433, People's Republic of China
} 